\title{
Numerical Simulation of Muti-Jet Ejector
}

\author{
Lin JIANG ${ }^{1}$ \\ School of Aeronautic Science and Engineering, Beihang \\ University, \\ Beijing 100191, P. R. China; \\ kfcllov@163.com
}

\begin{abstract}
The influence of nozzle location on the efficiency of ejector was discussed according to lots of calculated results. Changing nozzle location will have influence on the efficiency of ejector without modifying ejector structure.
\end{abstract}

\section{Keywords-ejector, efficiency, nozzle location , collision loss}

\section{INTRODUCTION}

For decades, ejectors have been studied for a large number of different applications in 2005 Bartosiewiczetal report about the ejectors have been used in aircraft propulsion systems for thrust augmentation purposes. And the research on ejectors used in high energy chemical lasers is conducted by Addy and Dutton in 1981.what's more, ASHRAE pointed out the utilization of ejectors in direct contact evaporative cooling applications used for drying medical drugs and food items.

Most of the current research on the internal flow of ejectors are consider as one-dimensional flow. And the momentum conservation is used to create equations. In most case, The structure of ejectors have been identified, only the specific size needs to be calculated. Accordingly, when the structure of ejector is uncertain, the method described above can't be used to calculate. What's more, because above method using onedimensional equations, the specific details on the ejector internal flow can't be give by this method

In this paper. We use the method of computational fluid dynamics, and the popular commercial CFD software--FLUENT to analyze the parameters and internal flow of Multijet Injector, In order to solve the insufficient of the traditional calculation method.

\section{THE FUNDAMENTAL OF EJECTOR}

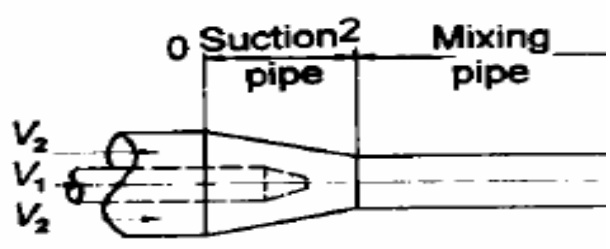

Figure 1. Schematic of the air ejector

The propellant gas from the nozzle with a certain velocity from the nozzle have collision momentum exchange with the Inhaled gas, so as to drive the gas move forward. Then the two

\author{
Song-ping $\mathrm{WU}^{1,2,3}$ \\ 2. National Laboratory for Computational Fluid Dynamics, \\ Beihang University, \\ Beijing 100191, P. R. China; \\ 3. Ministry of Education Key Laboratory of Fluid \\ Mechanics, Beihang University, \\ Beijing 100191, P.R. China
}

kind of gases are mixed within the mixing tube. In the mixing pipe, when the surface of the gas is pushed forward. the back of the gas becomes scarce and the pressure drop. cause a negative pressure near the outlet of the suction pipe and inlet of the mixing tube inlet section. So the Inhaled gas continue to be sucked into the mixing pipe, the greater the injection kinetic energy of the propellant gas have, the greater the pumping force will be. And more gas will be taken away.

\section{NUMERICAL SimULATION OF MUti-JET EJECTOR}

A the parameters of ejector

1) The amount of air sucked can measure by mass ejection coefficient

$$
u=\frac{m_{1}}{m_{2}}
$$

In the formula

$$
\begin{aligned}
& \mathrm{u} \text {-------mass ejection coefficient } \\
& m_{1} \text {-----Inhaled mass flow rate } \\
& m_{2} \text {-----injection mass flow rate } \\
& 2 \text { 2) Ejector efficiency }
\end{aligned}
$$

The ejector efficiency: the ratio of the effective work of the Inhaled gas obtained with the energy consumption of the injection gas.

$$
\begin{gathered}
E_{1}=\frac{P_{3}}{\rho_{3}} M_{1}+\frac{w_{3}^{2}}{2} M_{1}-\left(\frac{P_{1}}{\rho_{1}} M_{1}+\frac{w_{1}^{2}}{2} M_{1}\right) \\
E_{2}=\frac{P_{2}}{\rho_{2}} M_{2}+\frac{w_{1}^{2}}{2} M_{2}-\left(\frac{P_{3}}{\rho_{3}} M_{2}+\frac{w_{3}^{2}}{2} M_{2}\right) \\
\eta=\frac{E_{2}}{E_{1}} \times 100 \%
\end{gathered}
$$

$\eta$----- Ejection efficiency

$E_{1}$-------the effective work of the Inhaled gas obtained

$E_{2}$-------the energy consumption of the injection gas

$P_{1}$------Inlet Pressure $P_{2}$--------Ejection Pressure

$P_{3}$------Outlet Pressure 
$\rho_{1}$------Inlet Density $\rho_{2}$------Ejection Density

$\rho_{3}$------Outlet Density

$w_{1}$------Inlet Velocity $w_{2}$------Ejection Velocity

$w_{3}$------Outlet Velocity

$M_{1}$-----Inlet mass flow rate $M_{2}$-----Ejection mass flow rate

$M_{3}$-----Outlet mass flow rate

\section{B research object}

In this paper, compare to the traditional atmospheric ejector this Muti-jet Injector has 10 nozzles, and those nozzles are symmetrically distributed in both sides. The Inhaled gas and injection gas are ideal gas. the air pressure at the inlet or the outlet is standard atmospheric.

\section{Turbulence model and control equation}

In order to simplify the practical problems .Before establishing mathematical model. we consider the gas as ideal gas. the fluid is at room temperature.. the internal flow is Steady-state turbulence .The ejector wall satisfy the no-slip boundary conditions. In this paper, we use two-equation $\kappa-\varepsilon$ model to solve the ejector internal flow problems. $\kappa-\varepsilon$ model is widely used in scientific research and engineering practice, because of it's economic and reasonable accuracy.

the control equations of $\kappa-\varepsilon$ model, including the continuity equation, momentum equation, species equation $\kappa-\varepsilon$ equation is used to solve the turbulence problem.

\section{Computational grid and boundary conditions}

First, Using CATIA software to draw geometric model of the ejector. Then import the geometric model into ANSYS ICEM so as to generate the computational grid.

In this paper, the grid of the ejector is unstructured mesh, this kind of grid have geometric flexibility and better grid quality. It can also be able to deal with complex boundary problems and have adptive computing, reduce calculating work, improve the calculation accuracy.

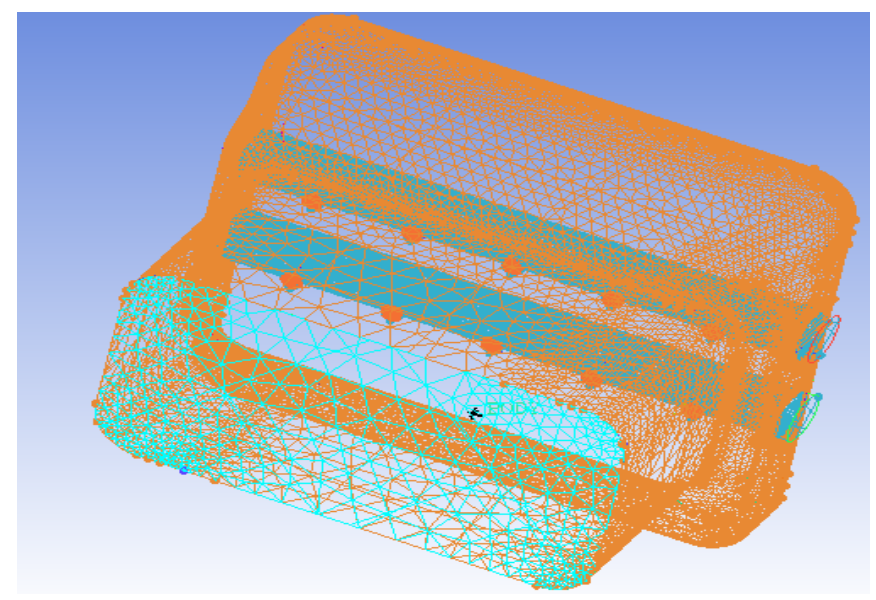

Figure 2. Grid of Muti-jet ejector

\section{E Analysis of simulation results}

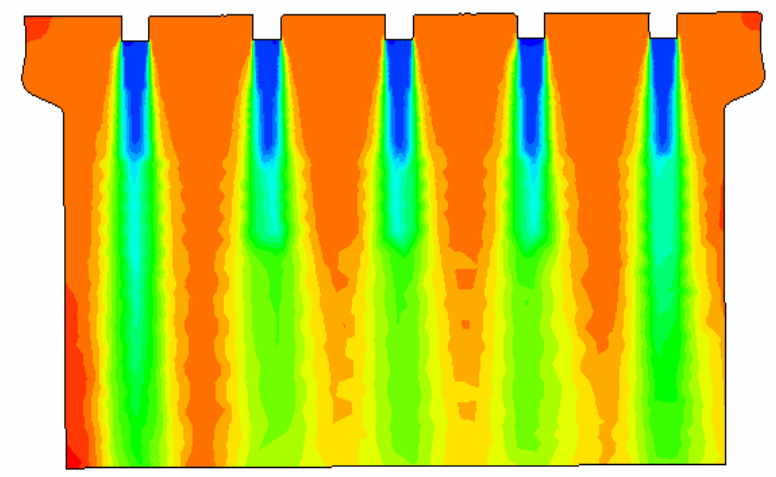

Figure 3. Velocity distribution

The picture above shows the Velocity distribution. Inhaled gas into the ejector tube with a certain speed, then have energy, momentum exchange with the injection gas in the mixing pipe. The Mixed gas flow out after mixing uniformly, and have a stabilized speed.

\section{F Factors affecting the ejector function}

1) The kinetic energy of the propellant gas

The greater the kinetic energy of the propellant gas has, the stronger the ability of the gas to be ejected, when the propellant gas driven Inhaled gas will generated collision loss, This loss will reduce the efficiency of the ejector. it is equal to the difference between the kinetic energy before and after the gas mixture (Represented by $\Delta \mathrm{E}$ )

$$
\Delta E=\frac{1}{2} \frac{m_{1} m_{2}}{m_{1}+m_{2}}\left(w_{1}-w_{2}\right)^{2}
$$

collision loss of unit flow injection gas unit flow

$$
\begin{aligned}
& \delta E_{1}=\frac{\Delta E}{m_{1}}=\frac{1}{2} \frac{m_{2}}{m_{1}+m_{2}}\left(w_{1}-w_{2}\right)^{2} \\
& \delta E_{2}=\frac{\Delta E}{m_{2}}=\frac{1}{2} \frac{m_{2}}{m_{1}+m_{2}}\left(w_{1}-w_{2}\right)^{2}
\end{aligned}
$$

Collision loss is proportional to the velocity difference of gas before mixing. If the Inhaled gas before mixing is stationary. The kinetic energy of propellant gas is relatively larger. the greater the difference in velocity and Collision loss. As shown in the following figure. 


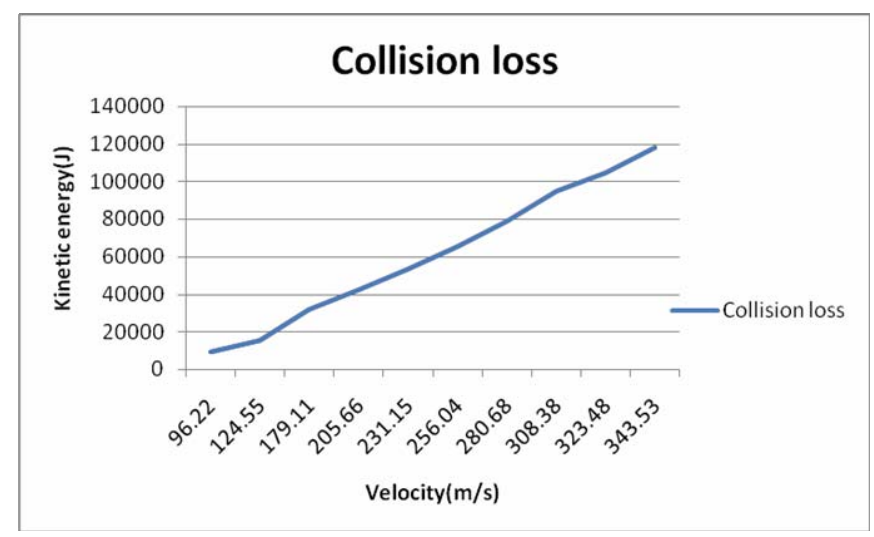

Figure 4. Collision loss

\section{2) Nozzle position of propellant gas}

The ratio of mixing tube sectional area $\mathrm{A} 1$ (diameter $\mathrm{d} 1$ ) and the ejection sectional area A2 (diameter d2) play a major role for the characteristics of the ejector. The suction pipe of the ejector is generally designed as cone. So the ejector nozzle is located just at the cone outlet, in order to increase the efficiency of the ejector. The value of A1/A2 will determine the size of the ejection coefficient $m_{1} / m_{2}$. So changing the relative position of the ejection nozzle is equal to change the value of $\mathrm{A} 1 / \mathrm{A} 2$, the increase of $\mathrm{A} 1 / \mathrm{A} 2$ will further improve the ejection efficiency

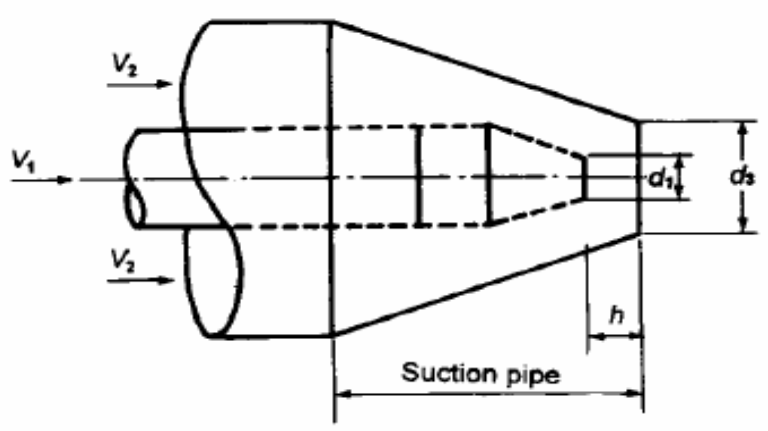

Figure 5. Relative location of ejected air nozzle in the suction pipe

Table.1 Effect of nozzle location on the ejector efficiency

\begin{tabular}{|c|c|c|c|c|}
\hline No. & $h(\mathrm{~mm})$ & $m_{1} / m_{2}$ & $\eta \mathbf{\%}$ & $\delta \eta \mathbf{0}$ \\
\hline 1 & 8 & 2.09 & 9.4 & 0 \\
\hline 2 & 13 & 2.24 & 12.6 & 34 \\
\hline 3 & 18 & 2.68 & 13.7 & 45.7 \\
\hline 4 & 23 & 2.26 & 12.4 & 31.9 \\
\hline 5 & 28 & 2.12 & 10.7 & 13.8 \\
\hline
\end{tabular}

$\delta \eta=\left\{\left(\eta_{i}-\eta_{1}\right) / \eta_{1}\right\} \times 100 \%(i=1,2,3,4,5)$

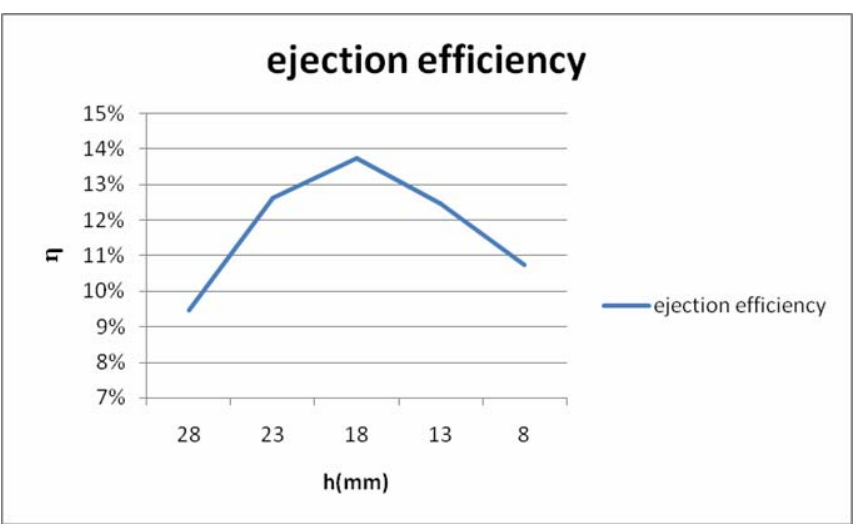

Figure 6. Ejection efficiency

The calculation results show that $\mathrm{h}$ may increase the ejector pumping force within a certain range. so that the suction amount of the propellant gas increased, thereby improving efficiency, as well as the efficiency is a maximum value. Compare to $\mathrm{h}=8$, the efficiency of $\mathrm{h}=18$ increase of $45 \%$ or more. when $\mathrm{h}$ is too large. the efficiency will decrease because of collision loss. And you can get a larger ejector efficiency, when $13<\mathrm{h}<23$.

\section{CONCLUSION}

(1) Collision loss is proportional to the velocity difference of gas before mixing 。 Reducing the velocity difference between the Inhaled gas and injection gas. Or increasing the velocity of the injection gas. Can reduce the collision loss when the gas mixing.

(2)Increasing h appropriately can increase the efficiency of the ejector。But when the increase is too large so they will decrease the ejection efficiency. According to the simulation results above. when $13<\mathrm{h}<23$, we can get the maximum value of the ejector efficiency .

\section{REFERENCES}

[1] sokolov E Y, zinger N M. Ejector. Moscow : Energy Press, 1970;8

[2] Charles Michael Moss, Effects of several Geometric Parameters on the performance of Mutiple Nozzle Eductor System, AdA047186,Sep,1977

[3] Wilson,A, E“Max.O.C.Design and control of Jet aeration System",U.S.Pat.Application, 1973

[4] YouChao-lin,"Numerical Simulation of Multi-jet Injecto.GAS\&HEAT,2010"

[5] Xing gui-ju, "The effective method of increasing of ejector efficiency used in metallurgical industry". ACTA METALLURGICA SINICA. 2000

[6] Wang suo-fang, Li li-guo. Performance analysis of a multi-nozzle ejector [J].Transactions of Nanjing University of Aeronautics and Astronautics, 1996

[7] Wang suo-fang,LI Li-guo.A study onsupersonic 6-nozzle ejector performance [J]. Journal of Aerospace Power,1996

[8] Zhao Jing-ye ,Sun Hou-jun.Basic Principles and Applications of Ejector.Journal of beijing institute of civil engineering and arc. 2000 